ISSN: 2526-7884

Editor: Prof. Dr. Marconi Freitas da Costa

E-mail: cbr@ufpe.br
Avaliação: Double blind review

Recebido: 17 de Fevereiro, 2021

Aceito: 13 de Abril, 2021

\title{
ANTECEDENTES E CONSEQUÊNCIAS DO BRAND HATE: UMA REVISÃO DA LITERATURA
}

\author{
Antecedents and Outcomes of Brand Hate: A Literature Review
}

\author{
Rémi Silva1 \\ ORCID: http://orcid.org/0000-0001-7007-0985 \\ E-mail: r.ssilva14@gmail.com \\ Suzanne Amaro ${ }^{1}$ \\ ORCID: http://orcid.org/0000-0001-9263-6951 \\ E-mail: samaro@estgv.ipv.pt
}

\section{${ }^{1}$ Instituto Politécnico de Viseu, Viseu, Portugal}

\begin{abstract}
Resumo
No Marketing, as emoções sentidas por uma pessoa em relação a uma marca são um aspeto de extrema importância, uma vez que afetam a forma como os consumidores se comportam em relação a uma marca. Em particular, as emoções e experiências negativas tendem a ser mais marcantes e são mais suscetíveis a serem divulgadas aos outros. Desta forma, é pertinente para as marcas estudarem o Brand Hate, ou seja, o ódio à marca. Este estudo teve como objetivo fazer uma revisão de literatura sobre os antecedentes e as consequências do Brand Hate. Apresenta, ainda, algumas indicações apontadas por autores para as marcas se reconciliarem com os consumidores e prevenirem o Brand Hate. Este estudo é, assim, de grande importância para as marcas atentarem da importância do Brand Hate,
\end{abstract}

\begin{abstract}
In Marketing, emotions consumers have for a brand are an extremely important aspect, since they affect the way consumers behave towards the brand. In particular, negative emotions and experiences tend to be more vivid and are more susceptible to being talked about with others. Thus, it is pertinent for brands to study Brand Hate. This study aimed to conduct a literature review on the antecedents and outcomes of Brand Hate. It also presents some indications pointed out by authors for brands to reconcile with consumers and prevent Brand Hate. Therefore, this study is of great relevance to brands to pay attention to the importance of Brand Hate, namely its harmful outcomes and its determinants.

Keywords: Brand Hate, Consumer Behaviour,
\end{abstract}


nomeadamente das consequências negativas e como é que ela poderá surgir.

Palavras-chave: Brand Hate, Comportamento do Consumidor, Emoções Negativas, Reconciliação, WOM Negativo.
Negative Emotions, Reconciliation, Negative WOM.

This work is licensed under a Creative Commons Attribution 4.0 International License.

\section{INTRODUÇÃO}

De uma forma geral, as emoções de carácter negativo têm um maior impacto nas pessoas do que as emoções de carácter positivo (Baumeister, Bratslavsky, Finkenauer, \& Vohs, 2001) e isso também acontece com os consumidores e o seu comportamento para com as marcas. As emoções negativas sentidas por uma marca têm influência nas ações do consumidor, tais como a apresentação de queixas ou a mudança de marca (Romani, Grappi, \& Dalli, 2012).

Vários investigadores têm se dedicado mais ao estudo das emoções positivas, nomeadamente o Brand Love, ou seja, o amor à marca, focando em aspetos como as suas causas e suas consequências (e.g. Albert \& Merunka, 2013; Albert, Merunka, \& Valette-Florence, 2009; Albert, Merunka, \& Valette-Florence, 2013; Bairrada, Coelho, \& Lizanets, 2019; Bairrada, Coelho, \& Coelho, 2018; Batra, Ahuvia, \& Bagozzi, 2012; Carroll \& Ahuvia, 2006; Fetscherin, 2014; Huber, Meyer, \& Schmid, 2015; Karjaluoto, Munnukka, \& Kiuru, 2016). No entanto, existe também o outro lado, quando ocorrem altos níveis de insatisfação para com uma marca, o Brand Hate, ou seja, o ódio à marca.

Em um estudo de Fetscherin (2019), os inquiridos foram questionados se sentiam emoções negativas por marcas e, no caso afirmativo, indicassem quais as marcas pelas quais tinham esse sentimento. Uma grande percentagem de consumidores (75\%) respondeu que tinham sentimentos negativos por marcas. Curiosamente, as marcas mais mencionadas neste estudo são ao mesmo tempo marcas que aparecem como as mais amadas, nomeadamente a Apple, Walmart, Nike e Mcdonalds.

Os acontecimentos que envolvem emoções negativas estão mais proeminentes na mente das pessoas que aqueles com emoções positivas (Baumeister et al., 2001), o que realça a importância de estudar as emoções negativas e as consequências que delas advém. 0 estudo das emoções negativas no consumidor, e como revertê-las, é ainda mais importante, devido à era digital que atravessamos, onde os consumidores têm acesso a mais informação, verídica ou não (Ahmed \& Hashim, 2018). Por outro lado, os consumidores têm um maior poder e influência através das redes sociais, onde podem expressar a sua opinião, principalmente a negativa (Ahmed \& Hashim, 2018). Através destes meios, é extremamente fácil criticar, fazer ataques às marcas e influenciar um número considerável de outros consumidores.

Pode-se, assim, concluir que o Brand Hate tem muita importância atualmente, no que diz respeito à temática do comportamento do consumidor. No entanto, investigação sobre o Brand Hate é escassa e negligenciada (Zarantonello, Romani, Grappi, \& Bagozzi, 2016; Zhang \& Laroche, 2020). Assim, este estudo tem como objetivo clarificar o que é, quais as suas causas e consequências e como é que as marcas devem agir. Os resultados são importantes para as marcas se aperceberem da importância que o Brand Hate tem, assim como das consequências negativas e como é que ela poderá surgir. Mais, o estudo de emoções negativas é uma vantagem para as marcas para se prevenirem de potenciais perdas, ao mesmo tempo que podem melhorar a qualidade dos seus serviços e o bem-estar dos consumidores (Zhang \& Laroche, 2020).

Para atingir os objetivos deste estudo, em Outubro de 2020 foram efetuadas pesquisas na base de dados Science Direct e Google Académico para selecionar artigos que abordassem o tema Brand Hate, usando como palavra chave Brand Hate. A utilização de base de dados para pesquisar artigos para revisões de literatura é uma metodologia muito utilizada em diversos estudos na área do Marketing (e.g. Javalgi \& La Toya, 2018; Misirlis \& Vlachopoulou, 2018). 


\section{REVISÃO DE LITERATURA \\ Brand Hate}

Em 2006, antes de o tema ser largamente desenvolvido, Carroll e Ahuvia (2006) argumentavam que o Brand Hate era o oposto a Brand Love, tema em que se focava o trabalho dos autores tendo proposto o Brand Hate como tema a ser investigado em futuros estudos. No entanto, não é assim tão claro que Brand Hate possa ser encarado simplesmente como o oposto de Brand Love e existe alguma controvérsia sobre este assunto (Kucuk, 2019a). De facto, o amor e o ódio não são sentimentos completamente opostos, até porque alguns estudos têm demonstrado que as pessoas podem sentir amor e ódio a uma marca, por razões diferentes (e.g. Zarantonello, Romani, Grappi, \& Fetscherin, 2018). Por outro lado, o facto do consumidor não sentir amor por uma marca, não significa que odeie essa marca (Kucuk, 2019a).

De acordo com Kucuk (2019a), Brand Hate pode ser definido como "um estado psicológico, através do qual o consumidor forma emoções intensamente negativas para com as marcas que atuam de forma defeituosa, dando aos consumidores experiências más e dolorosas" (p.20). O Brande Hate é, assim, um sentimento extremamente negativo e forte que um consumidor sente por uma marca (Bryson, Atwal, \& Hultén, 2013; Zhang \& Laroche, 2020), representando a forma mais intensa e extrema de não gostar de uma marca (Bryson et al., 2013; Romani et al., 2012; Zarantonello et al., 2016). Normalmente esse sentimento é acompanhado por outras emoções, nomeadamente raiva, tristeza e medo (Zhang \& Laroche, 2020).

É em torno dos vários tipos de emoções que autores têm conceptualizado o Brand Hate como um constructo multidimensional e têm defendido que poderá haver várias formas de odiar uma marca. Por exemplo, no estudo do Zarantonello et al. (2016), os autores verificaram que o Brand Hate poderia ser decomposto em dois componentes, o Brand Hate ativo e o Brand Hate passivo. 0 ativo inclui sentimentos como raiva e desgosto, enquanto o passivo tem associadas a si emoções como o medo, o desapontamento e a vergonha.

Fetscherin (2019), baseado em três emoções principais, a repugnância, o desprezo e a raiva, identifica cinco tipos de Brand Hate com base nas várias conjugações destas emoções: o Cool Hate (ódio calmo), o Hot Hate (ódio intenso), o Simmering Hate (ódio fervoroso), o Burning Hate (ódio fogoso) e o Boiling hate (ódio em ebulição). Também Kucuk (2019b) divide o Brande Hate em três níveis de intensidade. 0 mais intenso é o Burning Brand Hate, seguido de Boiling Brand Hate e, finalmente, o menos intenso, o Seething Brand Hate.

\section{Antecedentes de Brand Hate}

As causas para um consumidor sentir Brand Hate podem ser classificadas em duas grandes categorias: causas relacionadas com a marca e causas relacionadas com o consumidor (Kucuk, 2019a). As causas relacionadas com o produto, prendem-se essencialmente com experiências negativas que o consumidor teve com a marca, tais como falhas com produtos ou serviços, ou inclusive, com o próprio atendimento e comportamento da marca para com os mesmos (Hegner et al., 2017). A experiência negativa pode ser causada também pelo produto ou serviço não ter correspondido às expectativas (Joshi \& Yadav, 2020). Seja qual for a origem, as experiências negativas têm sido apontadas em vários estudos como uma das origens do Brand Hate (e.g. Hashim \& Kasana, 2019; Joshi \& Yadav, 2020; Zarantonello et al., 2016).

Zhang e Laroche (2020) salientam que não é só a experiência negativa que leva ao Brand Hate, são as experiências negativas recentes e as experiências negativas repetidas. Zarantonello et al. (2016) concluíram que quando os consumidores têm experiências negativas com as marcas, os níveis de Word of Mouth (WOM) negativo, queixas e protestos são mais elevados do que, por exemplo, quando apenas têm uma imagem negativa sobe a marca. Se a experiência negativa não for rapidamente recuperada pela marca, o consumidor vai-se sentir extremamente insatisfeito e frustrado e rapidamente isso poderá transformar-se em Brand Hate (Kucuk, 2019a).

O Brand Hate pode resultar da própria personalidade do consumidor (Kucuk, 2019a) ou então por existir uma desarmonia ou incompatibilidade entre as crenças e os gostos do consumidor e aquilo que a marca representa e comunica para com o seu público (Hegner et al., 2017). Neste caso, as causas não estão relacionadas com o produto, mas com o consumidor. 
Kucuk (2019b), usando o modelo de Personalidade Big Five, descobriu que pessoas com maior autoconfiança, e pessoas mais competitivas, podem sentir mais Brand Hate, agindo assim de forma mais intensa e agressiva que os restantes, levando a repercussões mais públicas e danosas para a marca. Por outro lado, pessoas menos confiantes preferem afastar-se das marcas que odeiam, optando por evitar a marca. Assim sendo, estes consumidores são menos expressivos sobre o seu ódio à marca e mais reservados nas suas críticas.

Esta distinção de diferentes comportamentos perante Brand Hate, baseada na personalidade das pessoas, é importante, pois, detetar previamente consumidores autoconfiantes e competitivos, pode ser usado como sinal de aviso e identificador de um possível consumidor suscetível a ter um alto Brand Hate pela marca (Kucuk, 2019b).

Kucuk (2019b) assinala ainda outro aspeto curioso, afirmando que o seu estudo não revelou qualquer resultado significativo no que diz respeito à relação entre a agressividade e o Brand Hate. Uma teoria explicativa que o mesmo aponta é que, pessoas com esse traço distintivo, tendem a enervar-se rapidamente, mas, por sua vez, também tendem a acalmar-se com igual rapidez. Desta forma, a agressividade não é um estado que perdure muito no tempo e, assim, acaba por sortir menos efeitos danosos do que seria de pensar.

Hegner et al. (2017) e Hashim e Kasana (2019) apontam ainda outras causas, relacionadas com a incompatibilidade ideológica entre consumidor e marca, seja a nível legal, social ou moral. Caso a marca atue em algum destes níveis, de forma contrária às ideologias do consumidor, tal pode levar a que sejam criados nos consumidores sentimentos negativos para com a marca.

Muito recentemente, Joshi e Yadav (2020) descobriram que os consumidores podem desenvolver ódio por uma marca por influência dos outros. Hashim e Kasana (2019) também verificaram que os rumores sobre uma marca poderiam conduzir ao Brand Hate. Estes resultados parecem ainda reforçar mais a importância da consequência WOM negativo, ou seja, quando uma pessoa odeia uma marca, poderá mesmo influenciar outros também a odiarem essa marca. Neste contexto, é crucial que as marcas desenvolvam esforços para evitar o Brand Hate, ou quando esse sentimento já estiver desenvolvido, procurar a reconciliação com o consumidor. A Tabela 1 resume os antecedentes do Brand Hate.

Tabela 1

Antecedentes do Brand Hate

\begin{tabular}{c|l|l}
\hline \multicolumn{1}{c|}{ Antecedentes } & \multicolumn{1}{|c}{ Autores } & \multicolumn{1}{c}{ Principais Resultados } \\
\hline Relacionadas com o Produto & \multicolumn{1}{|c}{} \\
\hline Experiências negativas com a marca & $\begin{array}{l}\text { Hashim e Kasana (2019) } \\
\text { Hegner et al. (2017) }\end{array}$ & $\begin{array}{l}\text { As experiências negativas com as } \\
\text { marcas (e.g. falhas, atendimento, não } \\
\text { correspondências às expetativas) } \\
\text { pode levar ao Brand Hate. }\end{array}$ \\
\hline
\end{tabular}

\begin{tabular}{c|l|l}
\hline Relacionadas com o Consumidor & & \\
\hline $\begin{array}{l}\text { Incompatibilidade ideológica entre } \\
\text { consumidor e marca }\end{array}$ & $\begin{array}{l}\text { Hashim e Kasana (2019) } \\
\text { Hegner et al. (2017) }\end{array}$ & $\begin{array}{l}\text { Consumidores podem sentir Brand } \\
\text { Hate por marcas que atuam de forma } \\
\text { contrária às suas ideologias. }\end{array}$ \\
\hline Personalidade do Consumidor & $\begin{array}{l}\text { Kucuk (2019a) } \\
\text { Kucuk (2019) }\end{array}$ & $\begin{array}{l}\text { Consumidores com auior } \\
\text { autoconfiança e competitivas são } \\
\text { mais suscetíveis de sentirem Brand } \\
\text { Hate. Uma personalidade agressiva } \\
\text { não tem influência. }\end{array}$ \\
\hline WOM negativo/influência dos outros & $\begin{array}{l}\text { Hashim e Kasana (2019) } \\
\text { Joshi e Yadav (2020) }\end{array}$ & $\begin{array}{l}\text { A influência dos outros pode levar } \\
\text { um consumidor a sentir Brand Hate, } \\
\text { mesmo sem ter tido uma experiência } \\
\text { negativa com a marca. }\end{array}$ \\
\hline
\end{tabular}




\section{Consequências do Brand Hate}

Quando um consumidor sente ódio à marca, poderá ter diferentes tipos de reações. As reações poderão ir desde evitar comprar a marca até comportamentos mais visíveis e danosos, que os consumidores insatisfeitos utilizam para demonstrar o seu descontentamento para com a marca (Bryson et al., 2013). Não há dúvida que qualquer que seja a reação do consumidor, será sempre prejudicial para a marca. Uma vez que existem vários tipos de Brand Hate, estudos têm demonstrado que a reação dos consumidores poderá depender do tipo de ódio que sentem pela marca (Fetscherin, 2019; Kucuk, 2019b).

Uma das principais reações do Brande Hate é que o consumidor vai evitar a marca (Hegner, Fetscherin, \& van Delzen, 2017). O desejo de evitar a marca é a necessidade que um cliente sente em cessar todos os contactos com a marca, de se afastar da mesma e de tudo o que essa possa representar (Grégoire, Tripp, \& Legoux, 2009), ou seja, rejeita deliberadamente uma marca (Lee, Motion, \& Conroy, 2009). De salientar que alguns autores utilizam este termo de forma indistinta com mudar de marca (e.g. Oliva, Oliver, \& MacMillan, 1992). Vários autores têm consistentemente corroborado que evitar a marca ou mudar de marca são consequências do Brand Hate (e.g. Fetscherin, 2019; Grégoire et al., 2009; Zarantonello et al., 2016). Os consumidores deixam de comprar a marca quando sentem ódio pela marca (Curina, Francioni, Hegner, \& Cioppi, 2020). Evitar a marca é uma das formas mais passivas do consumidor reagir (Grégoire et al., 2009).

De facto, existem outros comportamentos mais ativos, que podem ser mais prejudiciais para a marca. Por exemplo, Grégoire et al. (2009) indicam que uma consequência proveniente do Brand Hate é desejo de vingança. 0 desejo de vingança pode ser definido como o desejo que um cliente sente em punir e prejudicar a marca, de forma a magoá-la, em resposta ao mal que, previamente, poderá ter sido causado ao mesmo (Grégoire, Laufer, \& Tripp, 2010). Através da vingança, o consumidor tenta obter algum alívio dos sentimentos negativos que sente de uma forma que é normalmente espontânea e irracional (Zourrig, Chebat, \& Toffoli, 2009).

Os consumidores podem vingar-se da marca, de forma mais direta ou indireta. Segundo os autores, a vingança indireta ocorre fora da empresa. Este tipo de represálias dá-se através de WOM negativo sobre a marca em questão, para com os círculos de pessoas mais próximas daquele consumidor, como por exemplo, amigos e família ou o WOM realizado publicamente como, por exemplo em redes sociais, de forma a atingir e influenciar outras pessoas. Já a vingança direta implica uma represália mais direcionada à marca, num ambiente público. Os autores, dão como exemplo para este tipo de comportamento, os insultos diretos a representantes das marcas, que por norma dão a cara pelas mesmas ou casos mais extremos de consumidores que chegam a vandalizar propriedade da marca. 0 desejo de vingança poderá diminuir com o tempo, ao contrário do desejo de evitar a marca, que pode até aumentar ao longo do tempo, fazendo com que o dano seja irreversível (Grégoire et al., 2009).

Grégoire et al. (2010), afirmam, também, que os dois tipos de vingança abordados, direta e indireta, acabam por se traduzir em diferentes resultados, principalmente ao nível das implicações para a marca. Por exemplo, a vingança direta afeta fortemente os empregados que dão a cara pela marca, resultando numa grande pressão sobre os mesmos, o que pode dificultar a manutenção de um bom ambiente de trabalho e a preservação de uma alta moral e satisfação dos seus empregados. No entanto, a vingança direta pode ser uma vantagem para a marca, pois permite identificar os consumidores indignados (Grégoire et al., 2010). Tal é importante, pois, assim é possível tomar as devidas ações para reduzir ou mesmo erradicar esse sentimento do cliente em questão. Quando a reclamação acontece em privado, aos amigos e familiares, é quase impossível à marca ter conhecimento de tal comportamento até ser tarde de mais e perceber que perdeu o cliente (Grégoire et al., 2010). Torna-se mais difícil de controlar, de interromper a cadeia e de reverter esse sentimento (Grégoire et al., 2010). Ou seja, por vezes, é mais fácil recuperar um cliente que expresse a sua insatisfação de uma forma direta do que um que o faça de forma indireta.

Independentemente de ser feita de forma privada ou online, o WOM negativo é apontado por vários autores como uma das principais consequências do Brand Hate (e.g. Curina et al., 2020; Hegner et al., 2017; Jain \& Sharma, 2019; Joshi \& Yadav, 2020; Zarantonello et al., 2016). A este propósito, é 
importante referir que atualmente os consumidores queixam-se muito mais para além da esfera privada, devido à possibilidade de o fazerem publicamente online (Kucuk, 2019a). É importante notar que quando os consumidores se queixam de uma marca online, e se disserem que não voltam a comprar determinada marca, não voltam mesmo a comprar (Grégoire et al., 2009).

Uma última consequência apontada recentemente por Fetscherin (2019) é a disposição para fazer sacrifícios financeiros de forma a causar dano à marca (willingness to make financial sacrifices to hurt the brand). De facto, o autor encontrou que $44 \%$ dos respondentes estava disposto a fazer um sacrifício financeiro para prejudicar uma marca que odiassem. Este tipo de reação só acontece quando os consumidores sentem um determinado tipo de ódio, o Hot Hate, relacionado com a emoção de raiva. Um resumo das consequências de Brand Hate pode ser visualizado na Tabela 2.

Tabela 2

Consequências do Brand Hate

\begin{tabular}{|c|c|c|}
\hline Consequências & Autores & Principais Resultados \\
\hline $\begin{array}{l}\text { Evitar a } \\
\text { marca/Mudar de } \\
\text { Marca }\end{array}$ & $\begin{array}{l}\text { Curina et al. (2020); Hegner et al. (2017) } \\
\text { Fetscherin (2019); Grégoire et al. } \\
\text { (2009) } \\
\text { Zarantonello et al. (2016) }\end{array}$ & $\begin{array}{l}\text { O consumidor termina todos os contactos } \\
\text { com a marca que odeia e muda de marca. }\end{array}$ \\
\hline Desejo de vingança & $\begin{array}{l}\text { Grégoire et al. (2009); Grégoire et al. } \\
\text { (2010) } \\
\text { Zourrig et al. (2009) }\end{array}$ & $\begin{array}{l}\text { Um consumidor que sente ódio pela } \\
\text { marca procura a vingança de forma a } \\
\text { prejudicar a marca. }\end{array}$ \\
\hline WOM negativo & $\begin{array}{l}\text { Curina et al. (2020); Hegner et al. (2017) } \\
\text { Jain e Sharma (2019); Joshi e Yadav } \\
(2020) \\
\text { Zarantonello et al. (2016) }\end{array}$ & $\begin{array}{l}\text { O consumidor que sente Brand Hate irá } \\
\text { transmitir informações negativas sobre a } \\
\text { marca, quer online, quer offline. }\end{array}$ \\
\hline $\begin{array}{l}\text { Fazer sacrifícios } \\
\text { financeiros para } \\
\text { prejudicar a marca }\end{array}$ & Fetscherin (2019) & $\begin{array}{l}\text { Quando um consumidor sente Hot Hate, } \\
\text { um tipo de ódio relacionado com a raiva, } \\
\text { está disposto a gastar dinheiro para } \\
\text { causar danos à marca. }\end{array}$ \\
\hline
\end{tabular}

\section{Como evitar o Brand Hate e alcançar a reconciliação}

As marcas para se precaverem contra o Brand Hate devem dedicar mais atenção aos consumidores com traços de personalidade mais suscetíveis de desenvolverem esse sentimento, nomeadamente, a consumidores autoconfiantes e competitivos de forma a evitar possíveis conflitos no futuro, que escalem rapidamente até ao nível de Brand Hate (Kucuk, 2019b).

As marcas devem, também, desenvolver estratégias para quando acontecerem os primeiros problemas, conseguirem guiar o consumidor em direção ao desejo pela reconciliação com a marca, em vez de este seguir para o Brand Hate irreversível (Ahmed \& Hashim, 2018). 0 desejo de um consumidor se reconciliar com a marca irá depender muito da forma como a marca age com eles quando esta falha em alguma das variáveis prometidas ou acordadas (Ahmed \& Hashim, 2018).

De acordo com Kucuk (2019b) a marca tem de estar atenta às primeiras reclamações de um cliente e reagir logo, para prevenir que essa insatisfação se transforme em ódio. A captação de sinais de insatisfação pode ser com o recurso às novas tecnologias, através de ferramentas avançadas de escutar online (Kucuk, 2019a). Curina et al. (2020) recomenda que as empresas estejam atentas ao ambiente omnicanal, uma vez que os clientes insatisfeitos irão reclamar quer online, quer offline para mostrarem o seu desagrado. Uma vez detetados esses primeiros sinais de ódio, a marca necessita de desenvolver uma forma de comunicação eficaz para perceber as causas do ódio (Kucuk, 2019a). Se a marca não conseguir reagir logo na fase inicial Kucuk (2019a) considera que ainda existe uma segunda oportunidade, ainda que seja mais difícil e provavelmente mais dispendiosa para a marca. Se a marca não satisfizer o consumidor nessa segunda fase, aí as consequências vão ser muito piores para a marca, pois, existe uma grande probabilidade de o consumidor se tornar num Brand Hater irreversível, ou seja, um odioso da marca irreversível.

Kucuk (2019a) sugere, assim, três etapas para o processo de gestão do Brand Hate. Primeiramente, ouvir, de seguida, relacionar e, por último, negociar. De acordo com o autor, ouvir é o primeiro passo, de 
forma a explorar e entender as razões pelas quais o consumidor está revoltado ou desenvolveu sentimentos de Brand Hate. Relacionar é o segundo, envolvendo um pouco do primeiro passo, ouvir, mas adicionando momentos de perguntas e respostas ao cliente, fazendo-o entender que o representante daquela marca o está a entender e está interessado em compreender e resolver o problema. Por último, a negociação, sendo esta atingida através do segundo passo, relacionamento. Aqui pretende-se satisfazer os pedidos ou exigências do cliente, tendo em conta os interesses das empresas, de forma a chegar a um entendimento agradável para ambos os lados. A interação online com clientes insatisfeitos pode ser uma boa estratégia, pelo facto de exigir um menor investimento e ser possível dar uma resposta rápida ao cliente (Curina et al., 2020).

Assim, através das três fases idealizadas pelo autor para gerir o processo do Brand Hate, é possível a marca reduzir os efeitos dele, podendo mesmo levar a uma reconciliação pacífica com o consumidor. Ahmed e Hashim (2018), revelam também que muitas das vezes, os clientes não querem apenas uma compensação. Por vezes, estes esperam uma resposta respeitável, um pedido de desculpas e alguma atenção ao seu problema.

De acordo com Joireman, Grégoire, Devezer e Tripp (2013) uma estratégia efetiva é a combinação de um pedido de desculpas, juntamente com a oferta de algum tipo de compensação e uma explicação do sucedido, por parte da marca, aos seus consumidores para com os quais falhou, ao invés de aplicar esses fatores de forma individual. Segundo os autores, tal conjugação de medidas pode mudar a perceção negativa que os consumidores têm para com a marca naquele momento, conseguindo assim, pelo menos, neutralizar a emoção negativa do Brand Hate ou, até mesmo, conseguir transformar em sentimentos positivos, impedindo assim que este se afaste, retalie ou vingue da marca.

A Tabela 3 resume as principais estratégias para lidar com o Brand Hate sugeridas pelos autores nos últimos anos.

Tabela 3

Estratégias para Evitar Brand Hate

\begin{tabular}{|c|c|c|}
\hline Estratégias & Autores & Descrição da Estratégia \\
\hline \multicolumn{3}{|l|}{ Antecipação } \\
\hline Personalidade do Consumidor & Kucuk (2019a) & $\begin{array}{l}\text { As marcas devem dedicar mais atenção aos } \\
\text { consumidores com traços de personalidade mais } \\
\text { suscetíveis de desenvolverem Brand Hate. }\end{array}$ \\
\hline Sinais de insatisfação prévios & $\begin{array}{l}\text { Kucuk (2019a) } \\
\text { Curina et al. } \\
(2020)\end{array}$ & $\begin{array}{l}\text { A intervenção das marcas deve ser rápida. Para } \\
\text { tal, as marcas devem procurar sinais de } \\
\text { insatisfação prévios através de tecnologias e } \\
\text { também nas suas plataformas. }\end{array}$ \\
\hline \multicolumn{3}{|r|}{ 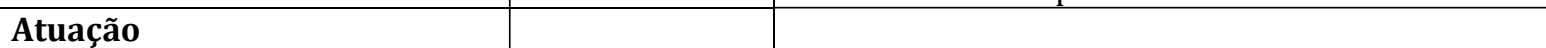 } \\
\hline $\begin{array}{ll}\text { Desenvolvimento } & \text { de } \\
\text { estratégias de reconciliação } & \end{array}$ & $\begin{array}{l}\text { Ahmed e } \\
\text { Hashim (2018) }\end{array}$ & $\begin{array}{l}\text { Devem ser desenvolvidas estratégias para guiar o } \\
\text { consumidor em direção à reconciliação. } 0 \text { desejo } \\
\text { de um consumidor se reconciliar com a marca } \\
\text { depende da forma como a marca age quando } \\
\text { ocorre uma falha. }\end{array}$ \\
\hline Oportunidades de atuação & Kucuk (2019a) & $\begin{array}{l}\text { A marca deve agir logo após a insatisfação. Tem } \\
\text { uma segunda oportunidade para o fazer, mais } \\
\text { dispendiosa e com maiores consequências. Após } \\
\text { essa fase, o consumidor poderá ficar a odiar a } \\
\text { marca de forma irreversível. }\end{array}$ \\
\hline Etapas da gestão do Brand Hate & $\begin{array}{l}\text { Kucuk }(2019 a) \\
\text { Curina et al. } \\
(2020)\end{array}$ & $\begin{array}{l}\text { Primeiramente a marca deve ouvir o consumidor, } \\
\text { de seguida deve relacionar-se com o mesmo e por } \\
\text { último negociar um entendimento satisfatório } \\
\text { para ambas as partes. }\end{array}$ \\
\hline Estratégia de reconciliação & $\begin{array}{l}\text { Ahmed e } \\
\text { Hashim (2018) } \\
\text { Joireman et al. } \\
\text { (2013) }\end{array}$ & $\begin{array}{l}\text { Por vezes o cliente não quer apenas } \\
\text { compensações ou explicações. Uma estratégia } \\
\text { eficaz consiste numa combinação de um pedido de } \\
\text { desculpas, com uma explicação para o sucedido, } \\
\text { seguido de uma compensação. }\end{array}$ \\
\hline
\end{tabular}




\section{CONCLUSÃO}

Este estudo teve como principal objetivo rever a literatura para identificar os antecedentes e as consequências do Brand Hate. Os principais determinantes de Brand Hate são as experiências negativas com a marca, a incompatibilidade ideológica entre consumidor e marca, a própria personalidade do consumidor e a influência dos outros. Estas emoções negativas para com uma marca podem causar problemas de diferentes níveis de gravidade às marcas, desde evitar a marca ao desejo de vingança. Desta forma, é extremamente importante que as marcas, primeiramente, estudem bem o seu mercado e o seu público-alvo, de forma a que possam comunicar corretamente para com o mesmo sem causar emoções negativas. Em segundo lugar, é também importante corresponder ou superar as expectativas que são criadas nos consumidores de forma a que não haja insatisfação, resultantes de produtos ou serviços com falhas. Por último, nunca descurar o consumidor, e oferecer a este toda a atenção necessária movendo todos os meios e estratégias necessárias de forma a reverter os sentimentos negativos do consumidor, pois um único consumidor pode levar à perda de muitos outros, através do muito influente WOM negativo. Para isso, as marcas devem estar preparadas e desenhar estratégias assentes principalmente em três fases, ouvir, relacionar e por fim negociar, de forma a reconciliar o consumidor com a marca. Quando o consumidor já manifesta Brand Hate, as marcas devem optar por a conjugação de um pedido de desculpas e uma compensação de forma a neutralizar ou reverter essas emoções.

Recomenda-se que, em futuros estudos sobre a temática, seja estudado como é que as diferentes gerações de consumidores reagem ao Brand Hate. Foi possível averiguar que as emoções têm um papel importante, mas seria interessante e de grande relevância perceber se essas emoções são encaradas de forma diferente entre gerações, causando comportamentos distintos no que diz respeito ao Brand Hate. Uma vez que as culturas afetam vários aspetos relacionados com o consumidor, outra linha de investigação poderia ser a comparação cultural do Brand Hate, quer a nível dos seus antecedentes e consequências, quer a nível de recuperação de consumidores que desenvolvem esse sentimento. Estes estudos seriam de enorme relevância para as marcas, pois permitiria que pudessem desenhar estratégias mais efetivas e concretas de neutralização e reversão dos sentimentos negativos associados à temática.

\section{Referências}

Ahmed, S., \& Hashim, S. (2018). The moderating effect of brand recovery on brand hate and desire for reconciliation: a PLS-MGA approach. International Journal of Business and Society, 19(3), 833850.

Albert, N., \& Merunka, D. (2013). The role of brand love in consumer-brand relationships. Journal of Consumer Marketing, 30(3), 258-266. doi:10.1108/07363761311328928

Albert, N., Merunka, D., \& Valette-Florence, P. (2009). The feeling of love toward a brand: Concept and measurement. Advances in Consumer Research, 36, 300 - 307

Albert, N., Merunka, D., \& Valette-Florence, P. (2013). Brand passion: Antecedents and consequences. Journal of Business Research, 66(7), 904-909. doi:10.1016/j.jbusres.2011.12.009

Bairrada, C. M., Coelho, A., \& Lizanets, V. (2019). The impact of brand personality on consumer behavior: the role of brand love. Journal of Fashion Marketing and Management: An International Journal, 23(1), 30-47. doi:10.1108/jfmm-07-2018-0091

Bairrada, C. M., Coelho, F., \& Coelho, A. (2018). Antecedents and outcomes of brand love: utilitarian and symbolic brand qualities. European Journal of Marketing, 52(3/4), 656-682. doi:10.1108/ejm02-2016-0081

Batra, R., Ahuvia, A., \& Bagozzi, R. P. (2012). Brand Love. Journal of marketing, 76(2), 1-16.

Baumeister, R. F., Bratslavsky, E., Finkenauer, C., \& Vohs, K. D. (2001). Bad is stronger than good. Review of General Psychology, 5(4), 323-370. doi:10.1037/1089-2680.5.4.323

Bryson, D., Atwal, G., \& Hultén, P. (2013). Towards the conceptualisation of the antecedents of extreme negative affect towards luxury brands. Qualitative Market Research: An International Journal, 16(4), 393-405. doi:10.1108/qmr-06-2013-0043 
Carroll, B. A., \& Ahuvia, A. C. (2006). Some antecedents and outcomes of brand love. Marketing Letters, 17(2), 79-89. doi:10.1007/s11002-006-4219-2

Curina, I., Francioni, B., Hegner, S. M., \& Cioppi, M. (2020). Brand hate and non-repurchase intention: A service context perspective in a cross-channel setting. Journal of Retailing and Consumer Services, 54, 102031. doi:10.1016/j.jretconser.2019.102031

Fetscherin, M. (2014). What type of relationship do we have with loved brands? Journal of Consumer Marketing, 31 (6/7), 430-440.

Fetscherin, M. (2019). The five types of brand hate: How they affect consumer behavior. Journal of Business Research, 101, 116-127. doi:10.1016/j.jbusres.2019.04.017

Grégoire, Y., Laufer, D., \& Tripp, T. M. (2010). A comprehensive model of customer direct and indirect revenge: understanding the effects of perceived greed and customer power. Journal of the Academy of Marketing Science, 38(6), 738-758. doi:10.1007/s11747-009-0186-5

Grégoire, Y., Tripp, T. M., \& Legoux, R. (2009). When Customer Love Turns into Lasting Hate: The Effects of Relationship Strength and Time on Customer Revenge and Avoidance. Journal of marketing, 73(6), 18-32.

Hashim, S., \& Kasana, S. (2019). Antecedents of brand hate in the fast food industry. Spanish Journal of Marketing - ESIC, 23(2), 227-248.

Hegner, S. M., Fetscherin, M., \& van Delzen, M. (2017). Determinants and outcomes of brand hate. Journal of Product \& Brand Management, 26(1), 13-25. doi:10.1108/jpbm-01-2016-1070

Huber, F., Meyer, F., \& Schmid, D. A. (2015). Brand love in progress - the interdependence of brand love antecedents in consideration of relationship duration. Journal of Product \& Brand Management, 24(6), 567-579. doi:10.1108/jpbm-08-2014-0682

Jain, K., \& Sharma, I. (2019). Negative outcomes of positive brand relationships. Journal of Consumer Marketing, 36(7), 986-1002. doi:https://doi.org/10.1108/JCM-07-2018-2764

Javalgi, R. G., \& La Toya, M. R. (2018). International marketing ethics: A literature review and research agenda. Journal of Business Ethics, 148(4), 703-720. doi:10.1007/s10551-015-2958-9

Joireman, J., Grégoire, Y., Devezer, B., \& Tripp, T. M. (2013). When do customers offer firms a "second chance" following a double deviation? The impact of inferred firm motives on customer revenge and reconciliation. Journal of Retailing, 89(3), 315-337. doi:10.1016/j.jretai.2013.03.002

Joshi, R., \& Yadav, R. (2020). Captivating Brand Hate Using Contemporary Metrics: A Structural Equation Modelling Approach. Vision: The Journal of Business Perspective, 097226291989217. doi:10.1177/0972262919892173

Karjaluoto, H., Munnukka, J., \& Kiuru, K. (2016). Brand love and positive word of mouth: the moderating effects of experience and price. Journal of Product \& Brand Management, 25(6), 527537. doi:10.1108/jpbm-03-2015-0834

Kucuk, S. U. (2019a). Brand hate: Navigating consumer negativity in the digital world (2nd ed.). Cham. Switzerland: Palgrave Macmillan.

Kucuk, S. U. (2019b). Consumer Brand Hate: Steam rolling whatever I see. Psychology \& Marketing, 36(5), 431-443. doi:10.1002/mar.21175

Lee, M. S. W., Motion, J., \& Conroy, D. (2009). Anti-consumption and brand avoidance. Journal of Business Research, 62(2), 169-180. doi:10.1016/j.jbusres.2008.01.024

Misirlis, N., \& Vlachopoulou, M. (2018). Social media metrics and analytics in marketing-S3M: A mapping literature review. International Journal of Information Management, 38(1), 270-276. doi.org/10.1016/j.ijinfomgt.2017.10.005

Oliva, T. A., Oliver, R. L., \& MacMillan, I. C. (1992). A catastrophe model for developing service satisfaction strategies. Journal of marketing, 56(3), 83-95.

Romani, S., Grappi, S., \& Dalli, D. (2012). Emotions that drive consumers away from brands: Measuring negative emotions toward brands and their behavioral effects. International Journal of Research in Marketing, 29(1), 55-67. doi:10.1016/j.ijresmar.2011.07.001

Zarantonello, L., Romani, S., Grappi, S., \& Bagozzi, R. P. (2016). Brand hate. Journal of Product \& Brand Management, 25(1), 11-25. doi:10.1108/jpbm-01-2015-0799

Zarantonello, L., Romani, S., Grappi, S., \& Fetscherin, M. (2018). Trajectories of brand hate. Journal of Brand Management, 25(6), 549-560. doi:10.1057/s41262-018-0105-5 
Zhang, C., \& Laroche, M. (2020). Brand hate: a multidimensional construct. Journal of Product \& Brand Management. doi:10.1108/JPBM-11-2018-2103]

Zourrig, H., Chebat, J.-C., \& Toffoli, R. (2009). Consumer revenge behavior: A cross-cultural perspective. Journal of Business Research, 62(10), 995-1001. doi:10.1016/j.jbusres.2008.08.006 